\title{
Miniaturized Planar Split-Ring Resonator Antenna
}

\author{
Kim, Oleksiy S.; Breinbjerg, Olav
}

Published in:

IEEE International Symposium on Antennas and Propagation

Link to article, DOI:

10.1109/APS.2009.5172040

Publication date:

2009

Document Version

Publisher's PDF, also known as Version of record

Link back to DTU Orbit

Citation (APA):

Kim, O. S., \& Breinbjerg, O. (2009). Miniaturized Planar Split-Ring Resonator Antenna. In IEEE International Symposium on Antennas and Propagation (pp. 1-4). IEEE. https://doi.org/10.1109/APS.2009.5172040

\section{General rights}

Copyright and moral rights for the publications made accessible in the public portal are retained by the authors and/or other copyright owners and it is a condition of accessing publications that users recognise and abide by the legal requirements associated with these rights.

- Users may download and print one copy of any publication from the public portal for the purpose of private study or research.

- You may not further distribute the material or use it for any profit-making activity or commercial gain

- You may freely distribute the URL identifying the publication in the public portal

If you believe that this document breaches copyright please contact us providing details, and we will remove access to the work immediately and investigate your claim. 


\title{
Miniaturized Planar Split-Ring Resonator Antenna
}

\author{
Oleksiy S. Kim* and Olav Breinbjerg \\ lectromagnetic Systems \\ Department of lectrical ngineering, Technical University of Denmark \\ Ørsted Plads, Building 8, DK- 8 Kgs. Lyngby, Denmark \\ -mail: osk@elektro.dtu.dk
}

\section{Introduction}

A split-ring resonator (SRR) is one of the electrically smallest resonant elements available at microwaves. Its applications range from compact filters to advanced metamaterials. Besides this, an electrically small antenna can be built using an SRR as it was recently shown in , where a broadside coupled SRR was excited by an arc-shaped monopole on a metal ground plane. The size of this self-resonant SRR-based antenna was demonstrated to be as small as $\lambda / \quad(k a \simeq . \quad)$, where $\lambda$ is the free space wavelength, $k$ is the wave number, and $a$ is a radius of a minimum sphere enclosing the antenna. As it is argued in , an antenna with $k a<$. can be considered as an electrically small antenna. Then, a planar SRR-based antenna presented in this paper can be regarded as an electrically very small antenna since it has $k a<$.

\section{Geometry and properties of the antenna}

A planar SRR-based antenna is composed of a broadside coupled SRR and an excitation dipole with arc-shaped arms surrounding the SRR (Fig. ). Both the SRR and the dipole are printed on the same substrate. The broadside coupled version of SRR is chosen due to its lower $R_{\text {ext }} / \lambda$ ratio as compared to the edge coupled counterpart . The antenna can be approximated by a parallel $R L C$ circuit tuned to a resonance with a series connected small capacitor. The parallel $R L C$ circuit and the tuning capacitor correspond to the SRR and the dipole, respectively. By varying the length of the dipole arms, which is equivalent to changing the reactance of the tuning capacitor, the antenna input impedance is tuned in a wide range.

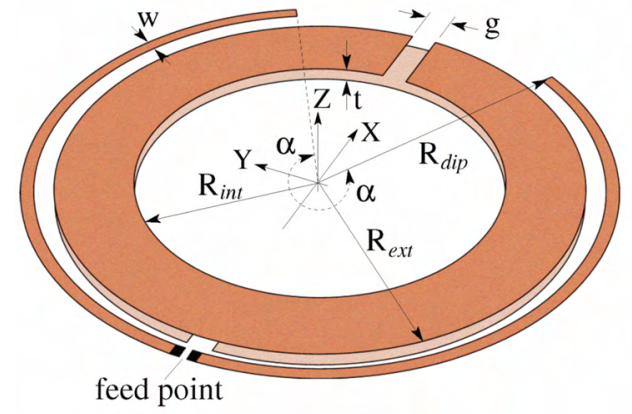

Figure : Antenna geometry (a)

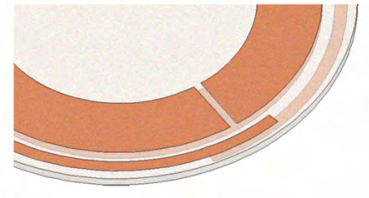

(b)

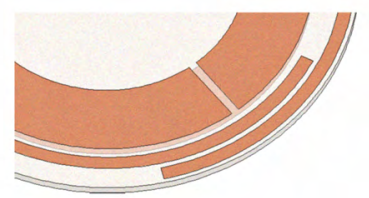

Figure : Broadside (a) and edge (b) overlapping dipole arms

As shown in , the input resistance at the resonance is inverse proportional to the dipole arm length, while the resonance frequency as well as the size of the antenna 
are governed by the SRR geometry. Reduction of the antenna electrical size can be achieved by using thin substrates with high dielectric permittivity. However, as the SRR gets smaller the total arc length of the curved dipole arms required to tune the antenna to a desired input impedance starts exceeding the perimeter of the SRR. To maintain the required dipole arm length it should either be moved away from the SRR, which increases the overall antenna diameter, or the arms overlap. In the later case there are two options:

- design 1: arms are printed on the opposite sides of the substrate (Fig. a);

- design 2: arms are printed on the same side of the substrate (Fig. b).

Simulations performed with the Ansoft's HFSS reveal that these two configurations demonstrate different characteristics.

\section{Numerical and Experimental Results}

The geometry with following parameters is investigated: $R_{\text {ext }} \quad .6 \mathrm{~mm}, R_{\text {int }}$ 6. $\mathrm{mm}, R_{\text {dip }} \quad \mathrm{mm}, t \quad$. $\mathrm{mm}, g \quad$. $\mathrm{mm}$, and $w \quad .6 \mathrm{~mm}$. The Rogers substrate with relative dielectric permittivity $\varepsilon$. is utilized. The conductivity of metalization is assumed to be $.8 \cdot{ }^{8} \mathrm{~S} / \mathrm{m}$. The antenna is directly fed by a -ohm coaxial feed line, whose effect is subsequently removed by deembedding the input impedance to the antenna feed point. The antenna designs and are simulated for a range of dipole arm lengths, and the results are summarized in Fig. , where the antenna resonance frequency and the input resistance at the resonance are plotted as functions of the angle $\alpha$. It is observed that with the non-overlapping dipole arms $\left(\alpha<8^{\circ}\right)$ both antenna designs resonate at the same frequency, and this frequency is nearly independent on the angle $\alpha$. However, as the arms overlaps $\left(\alpha>8^{\circ}\right)$ the curves start diverging and the resonance frequency starts varying with the angle $\alpha$. This is caused by a parasitic capacitor formed between the overlapping dipole arms. The parasitic capacitance is much smaller in the design and therefore the frequency variation is also weaker. Besides this, both designs yield a wide dynamic range in terms of input resistances the antenna can be matched to simply by varying the dipole arm length.

Since the design exhibits weaker dependence of the resonance frequency and the input resistance on the dipole arm length and consequently weaker sensitivity to numerical and manufacturing errors, it is preferable from the practical point of view. Moreover, it allows tuning functions with respect to the resonance frequency and input resistance to be rather independent on each other. Thus, the design was selected for experimental testing. To minimize the diameter of the antenna prototype the dipole width in the overlapping domain was set to $w$. mm. With the clearance of . $\mathrm{mm}$ added between the dipole arms and the substrate edge the overall diameter of the antenna is $.6 \mathrm{~mm}$ (Fig. ), which at $\mathrm{MHz}$ corresponds to $k a \simeq$. .

According to Fig. , the ohms input resistance at the resonance corresponds to the angle $\alpha \quad{ }^{\circ}$. The prototype, however, was fabricated with little longer arms $\left(\begin{array}{ll}\alpha & \circ\end{array}\right)$. By cutting the dipole arms from $\alpha{ }^{\circ}$ to $\alpha{ }^{\circ}$ the input resistance at the resonance was tuned from ohms to . ohms. Simulated and 
measured reflection coefficients are compared in Fig. . The relative error for both the angle $\alpha$ and the resonance frequency is less than $\%$, which is within margins set by tolerances on the relative permittivity and thickness of the utilized substrate.

Radiation measurements were performed at the DTU- SA Spherical Near-field Antenna Test Facility and the results are presented in Fig. 6 . The radiation pattern corresponds to that of a Z-directed magnetic dipole. Simulated and measured antenna parameters - angle $\alpha$, resonance frequency $f_{0}$, input resistance at the resonance $R_{0}$, bandwidth at - $\mathrm{dB} \Delta f_{-10 d B}$, quality factor $Q$ calculated using , eq.(86), peak directivity $D$, and efficiency $\eta$ - are summarized on Table .

\section{Conclusions}

A miniaturized planar antenna based on a broadside-coupled split ring resonator excited by an arc-shaped dipole is presented. The excitation dipole acts as a small tuning capacitor in series with a parallel $R L C$ circuit represented by the SRR. The antenna resonance frequency and dimensions a essentially determined by the SRR, while by varying the dipole arm length the input resistance is changed in a wide range, thus matching the antenna to a feed line and compensating for simulation and manufacturing inaccuracies. No additional matching network is required. Theoretically, there is no limit on how small this antenna can be. In practice, the lower bound is set by losses in utilized materials and manufacturing inaccuracies.

As an example, an antenna of $k a \simeq$. was designed, fabricated and tested. Although the initially fabricated antenna prototype had the input impedance of

ohms, it was subsequently tuned to ohms simply by cutting out the excessive arm length. This tuning technique is especially useful in practical applications, since it allows the antenna to be tuned in-place and thereby compensate for various inaccuracies as well as for an antenna environment.

\section{Acknowledgements}

This work is supported by the Danish Research Council for Technology and Production Sciences within the TopAnt project (http://www.topant.dtu.dk). Dr. S. Pivnenko is acknowledged for the antenna radiation measurements.

\section{References}

O. S. Kim and O. Breinbjerg, "Miniaturized self-resonant split-ring resonator antenna," accepted for publication in Electronics Letters,

S. Best, "A discussion on the properties of electrically small self-resonant wire antennas," IEEE Antennas Propag. Magazine, vol. 6, no. 6, pp. - , .

R. Marques, F. Mesa, J. Martel, and F. Medina, "Comparative analysis of edgeand broadside- coupled split ring resonators for metamaterial design - theory and experiments," IEEE Trans. Antennas Propagat., vol. , no. , pp. $7-8$, 
A. Yaghjian and S. Best, "Impedance, bandwidth, and Q of antennas," IEEE Trans. Antennas Propagat., vol. , no. , pp. 8-

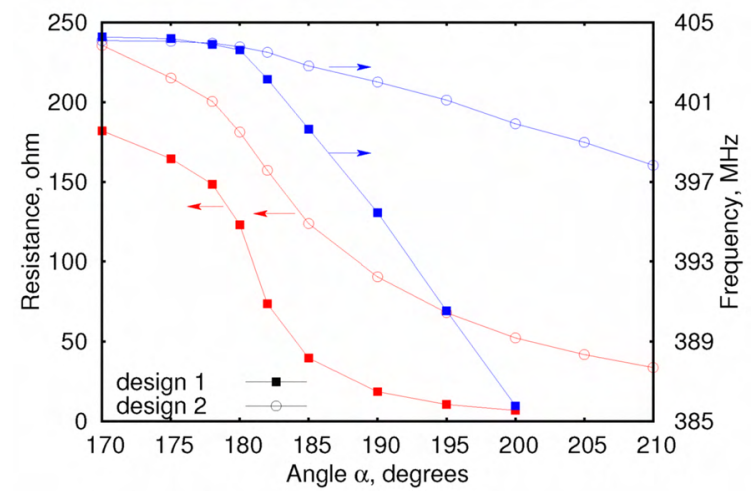

Figure : Input resistance and resonance frequency vs. dipole arm length.

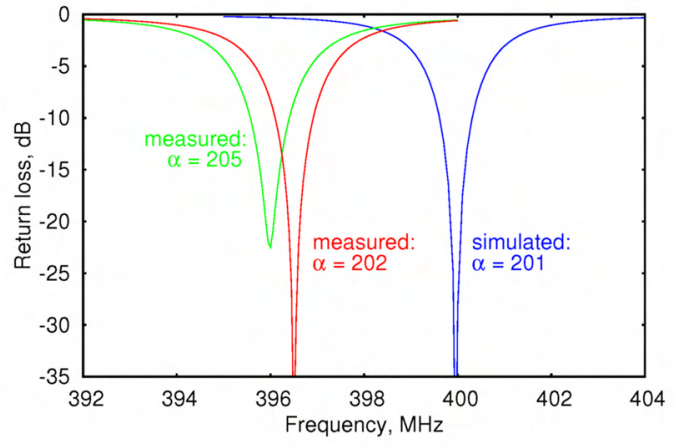

Figure : Simulated and measured return Figure 6: Measured radiation pattern loss

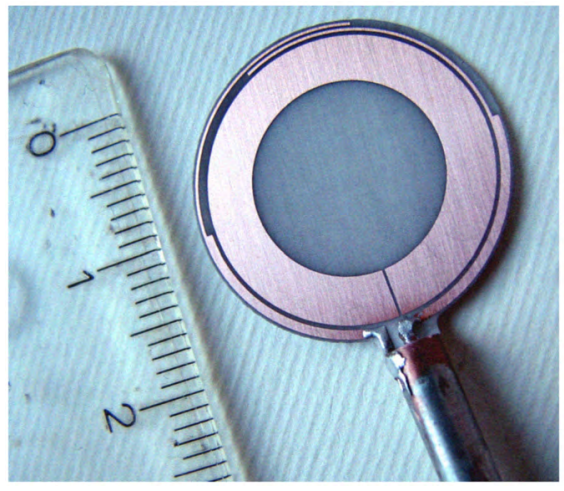

Figure : Fabricated antenna prototype (Design ).

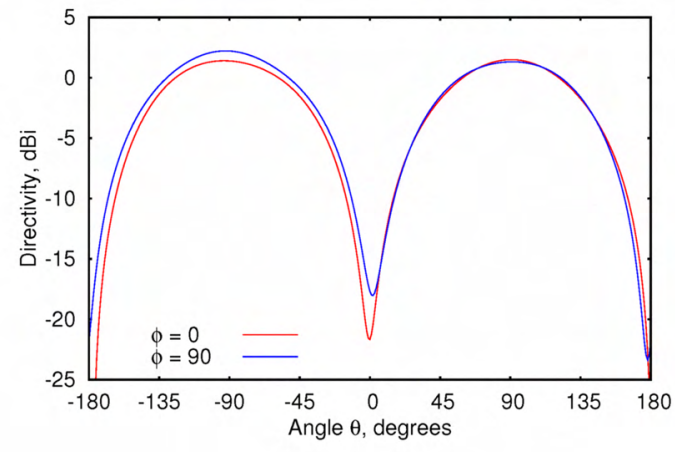

Table : Simulated and measured antenna parameters

\begin{tabular}{c|c|c|c|c|c|c|c}
\hline & $\alpha$ & $f_{0}, \mathrm{MHz}$ & $R_{0}, \mathrm{ohms}$ & $\Delta f_{-10 d B}, \mathrm{MHz}$ & $\mathrm{Q}$ & $D, \mathrm{dBi}$ & $\eta, \%$ \\
\hline simulated & $\circ$ &. & & .7 & &. & .8 \\
measured & $\circ$ & 6. &. & .8 & 8 &.. \pm &.. \pm \\
\hline
\end{tabular}

